\title{
Significant Increases in EEG Anterior-Posterior Alpha and Beta Powers By Transcranial Photobiomodulation (tPBM) in Healthy Humans With Exclusion of Thermal Effects
}

\section{Hashini Wanniarachchi}

The University of Texas at Arlington

\section{Xinlong Wang}

The University of Texas at Arlington

\section{Anqi Wu}

The University of Texas at Arlington

\section{F. Gonzalez-Lima}

The University of Texas at Austin

Hanli Liu ( $\nabla$ hanli@uta.edu )

The University of Texas at Arlington

\section{Research Article}

Keywords: transcranial photobiomodulation, tPBM, EEG, thermal stimulation, brain oscillations

Posted Date: March 31st, 2021

DOI: https://doi.org/10.21203/rs.3.rs-359452/v1

License: (c) (1) This work is licensed under a Creative Commons Attribution 4.0 International License.

Read Full License 

powers by transcranial photobiomodulation (tPBM) in healthy humans with exclusion of thermal effects

4 Hashini Wanniarachchi ${ }^{1}$, Xinlong Wang', Anqi Wu' ${ }^{1}$, F. Gonzalez-Lima ${ }^{2}$, Hanli Liu ${ }^{*}$

5 1. Department of Bioengineering, University of Texas at Arlington, Arlington, TX

6 2. University of Texas at Austin, Department of Psychology and Institute for Neuroscience, 7 Austin, TX

$8 \quad *$ Corresponding author:

$9 \quad$ Hanli Liu, Ph.D.

10 Email: hanli@uta.edu 
12 Transcranial photobiomodulation (tPBM) of the prefrontal cortex can improve human cognition 13 and increase electroencephalogram (EEG) alpha and beta powers, but it was unclear whether 14 tPBM-induced heat would influence EEG oscillation powers. This study aimed to prove that tPBM-induced increases in anterior-posterior EEG powers at alpha and beta bands would be significant after removal of tPBM-associated thermal effects. We performed both sham-controlled tPBM and sham-controlled thermal stimulation (thermo_stim) experiments under the eyes-closed resting state with concurrent recordings of 64-channel EEG before, during, and after 8-min tPBM at 1064-nm wavelength and thermo_stim with temperature from 33 to $41^{\circ} \mathrm{C}$, respectively, from healthy humans ( $n=46$ for tPBM; $n=14$ for thermo_stim). Sham-subtracted topographies of EEG powers at five frequency bands were averaged at the group level during and post both stimulations.

22 Two-sample t-tests with FDR correction and effect size were calculated for comparing tPBM and thermal effects at all five frequency bands. Right-frontal tPBM induced significant increases in EEG anterior-posterior alpha and beta powers under the eyes-closed conditions, consistent with the results previously reported in the eyes-open tPBM experiments. In contrast, right-frontal thermal stimulation under the eyes-closed resting state resulted in opposite effects on EEG power patterns with respect to those by $\mathrm{APBM}$. tPBM-induced enhancement in alpha and beta oscillations occurred during the 8-min intervention after exclusion of thermal effects. The ability of tPBM to synchronize alpha and beta oscillations in the anterior-posterior regions may be linked to the enhancement of frontoparietal network and the improvement of human cognition.

Keywords: transcranial photobiomodulation, tPBM, EEG, thermal stimulation, brain oscillations. 


\section{1. Introduction}

35 Photobiomodulation (PBM), also known as low-level laser therapy (LLLT) in clinical applications,

36 utilizes red to near-infrared (NIR) light to stimulate mitochondrial respiration in a wide range of

37 cells and tissues in the human body ${ }^{1-4}$. Transcranial photobiomodulation (tPBM) is a type of PBM

38 that delivers NIR light/laser to the human brain, which has shown promising outcomes in treating

39 psychiatric and neurological disorders ${ }^{5}$, such as depression and anxiety ${ }^{6}$, and traumatic brain

40 injuries ${ }^{7,8}$. Recent studies have reported that tPBM with a 1064-nm laser can enhance human

41 cognitive performance on a variety of cognitive tasks using sham-controlled experiments ${ }^{9-13}$.

42 Furthermore, we have recently demonstrated that 1064-nm tPBM enabled significant upregulation

43 in concentrations of hemoglobin oxygenation ([HbO]) and oxidized cytochrome-c-oxidase

44 ([CCO]) during and after tPBM on the human right forehead with high reproducibility and

45 robustness ${ }^{14-16}$. These findings supported and validated the hypothesized mechanism of action that

46 tPBM can photo-oxidize CCO, the key mitochondrial enzyme for cellular oxygen metabolism, to

47 boost the metabolic activities of cells ${ }^{17}$, especially neurons ${ }^{1,18}$. In addition, we investigated the

48 thermal impact of tPBM on measured alterations in $[\mathrm{HbO}]$ and $[\mathrm{CCO}]$, confirming that the heat-

49 induced warm sensation on the forehead would not give rise to the same increases in [HbO] and

$50 \quad[\mathrm{CCO}]$ as those seen by $\mathrm{tPBM}^{19}$.

51 There has been much less understanding and observation of electrophysiological responses to

52 tPBM in the human brain. Our recent results revealed that $\mathrm{PBM}$ is effective in enhancing the EEG

53 powers of large-scale alpha and beta oscillations in the human brain during eyes-opened resting

54 state, measured by 64-channel scalp EEG from healthy human subjects ${ }^{20,21}$. Similar observations

55 on EEG responses to tPBM were reported by other groups while using different experimental 
56 protocols ${ }^{22-24}$. All these studies consistently indicated that tPBM can also modulate neuronal or

57 electrophysiological synchronization and connectivity in the human brain.

It is expected and often experienced that a sizeable optical beam used for tPBM may produce

59 a warm sensation on the subject's forehead. Since the scalp EEG signal is sensitive to ambient

60 temperature or thermal stimulation of the subject's head ${ }^{25}$, the warmness created by the light/laser

61 illumination during $\mathrm{PBBM}$ can potentially affect or contaminate the net EEG signal induced only

62 by tPBM. Thus, an essential reservation or question on the action mechanism of tPBM would be

63 whether the improvement of behavioral performances and EEG power alteration might result from,

64 at least partially, thermal effects caused by light or laser illumination delivered on the subject's

65 forehead. Furthermore, it is known that there exist distinct temporal and spatial patterns in human

66 EEG signals between eyes-open and eyes-closed resting conditions ${ }^{26}$. EEG signals are more

67 vulnerable and sensitive to eye-blinks and drowsiness in the eyes-open resting state than in the

68 eyes-closed resting state. This raised another question on whether TPBM-induced power changes

69 of brain oscillation would be different between eyes-open and eyes-closed human resting state.

70 To address the two questions given above, we hypothesized that tPBM-induced increases in

71 anterior-posterior EEG powers at alpha and beta bands remained statistically significant during the

72 eyes-closed resting state, similar to those during the eyes-open resting state, after removal of

73 tPBM-associated thermal effects. Using the same experimental protocol as our previous studies

$74[3,7,11,15]$, we performed both sham-controlled tPBM and sham-controlled thermal stimulation

75 (thermo_stim) experiments. Specifically, we recorded 64-channel EEG before, during, and after

76 8-min tPBM and thermo_stim, respectively, from healthy human subjects ( $\mathrm{n}=46$ for tPBM; $\mathrm{n}=14$

77 for thermo_stim) under the eyes-closed resting state. Alterations in EEG power at five frequency

78 bands (delta, theta, alpha, beta, and gamma) and topographical patterns induced by each of tPBM 
79 and thermo_stim were then computed and compared. All these observations allowed us to prove

80 our hypothesis, as progressively presented in the following sub-sections.

\section{2. Materials and Methods}

\section{2.1. Participants}

83 For the sham-controlled tPBM experiment, 49 healthy human subjects (30 males, 19 females; 26

$84 \pm 8.8$ years of age) were enrolled from the local community of the University of Texas at Arlington.

85 However, 3 subjects were removed from the dataset due to self-reported/observed tiredness and/or

86 sleepiness during the experiments. For the sham-controlled thermo_stim experiment, another

87 group of 14 human subjects ( 8 males, and 6 females; $29 \pm 8.8$ years of age) were recruited from

88 the same community. No substantial age difference between the two gender groups (with a two-

89 tailed t-test, $p>0.1$ ) was observed. Exclusion criteria of participants: (1) previous diagnosis with

90 a psychiatric disorder, (2) history of neurological disease, (3) history of severe brain injury, (4)

91 history of violent behavior, (5) prior institutionalization or imprisonment, (6) current intake of any

92 psychotropic medicine or drug, (7) history of smoking, (8) excessive alcohol consumption, (9)

93 pregnancy, and (10) previous diagnosis with diabetes as required by the manufacturer of the laser

94 (Cell Gen Therapeutics LLC, Dallas, Texas). All the participants were told to avoid any caffeine

95 beverages 2-3 hours before each experiment. All experimental procedures were approved by the

96 Institutional Review Board of the University of Texas at Arlington; all methods were performed

97 in accordance with the relevant guidelines and regulations. Informed consent was obtained from

98 each participant prior to all experiments.

\section{2.2. Experimental Setup}

100 As shown in Fig. 1(a), a continuous-wave (CW) 1064-nm laser (Model CG-5000 Laser, Cell Gen

101 Therapeutics LLC, Dallas, TX, USA), cleared by the Food and Drug Administration (FDA), was 
102 utilized to conduct tPBM and the corresponding sham experiment in this study. This was the same

103 device used in our previous studies ${ }^{11,19,20,27}$. This laser was able to deliver a collimated beam in a

104 diameter of $4.2 \mathrm{~cm}$. We conducted tPBM with a total power of $3.5 \mathrm{~W}$, which led to a power density

105 of $\sim 0.25 \mathrm{~W} / \mathrm{cm}^{2}$ and a total energy dose of $1680 \mathrm{~J}$ over 8 min of tPBM $(3.5 \mathrm{~W} \times 480 \mathrm{~s}=1680 \mathrm{~J})$

106 on the right forehead, as indicated in Fig. 1(b). For the sham experiment, the laser device was on

107 but set to be $0.1 \mathrm{~W}$ during the 8-min stimulation time. In addition, a black colored cap was used to

108 block the laser aperture. As a result, the power density under the sham stimulation was further

109 confirmed to be $0 \mathrm{~W} / \mathrm{cm}^{2}$ by a sensitive power meter (Model 834-R, Newport Corp., Andover,

110 MA, USA) to ensure the complete impediment of laser light. The participants were instructed to

111 maintain their eyes closed with a minimal level of motion during the experiment. Subjects were

112 also directed to give minor hand gestures in response to the experiment operator for verifying that

113 they were not asleep during the experiment. At the end of the experiment, each participant was

114 asked to confirm that he/she was awake without much drowsiness and sleepiness during the entire

115 experimental period.

116 A Biosemi (64-channel) 10-10 EEG system was employed for data collection. Before each

117 experiment, electrical gel was applied on each electrode to improve the conductivity and signal to

118 noise ratio of the data acquisition. The stimulation protocol (see Fig. 1(c)) consisted of a 2-min

119 baseline, an 8-min tPBM, and a 3-min recovery period. In both sham and active tPBM experiments,

120 the laser aperture was pointing at the right forehead near the electrode locations of FP2 and AF8.

121 Both the participant and operator wore protection goggles to prevent any stray laser light from

122 entering their eyes. While the EEG data were acquired at either $256 \mathrm{~Hz}$ or $512 \mathrm{~Hz}$, all the $512 \mathrm{~Hz}$

123 data were down sampled to $256 \mathrm{~Hz}$ during data preprocessing for consistency. 

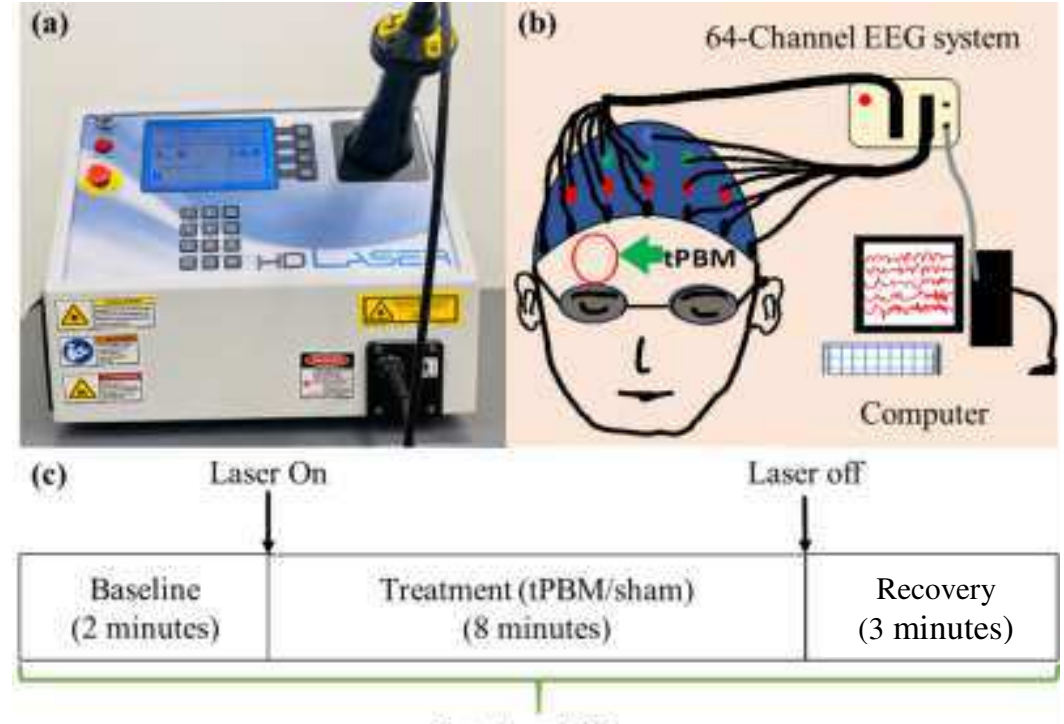
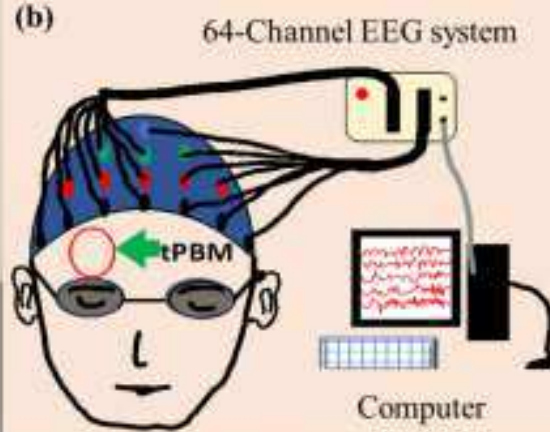

ata Acquisition replicate and induce the thermal stimulation on the human forehead through an ATS mode probe. 142 The ATS thermode can deliver temperatures ranging from $0{ }^{\circ} \mathrm{C}$ to $55^{\circ} \mathrm{C}$ with a maximum rate of

Fig. 1 Experimental setup for concurrent tPBM and EEG experiment. (a) Photo of FDA-cleared, 1064-nm, continuous-wave laser (Model CG-500). (b) Schematic diagram of the experimental setup: 64 electrodes of an EEG device were attached to an international 10-10 standard EEG cap, while the subject was wearing a pair of safety goggles and retained in eye-closed resting state during the sham or active tPBM condition. The laser aperture was pointed at the right forehead of the subject with 2-cm away from the skin. (c) Shows the experimental protocol including a 2-min baseline, 8-min stimulation (with a laser power of $3.5 \mathrm{~W}$ for tPBM and $0 \mathrm{~W}$ for sham), and a 3-min recovery period.

Designed as a single-blind, cross-over study, each subject took both sham and active tPBM experiments within a period of 1 week, with a minimum of 3 days between the two experiments.

The order of the sham or tPBM experiment was randomly assigned. All subjects were inquired about their experience after each experiment, including the heat sensation and potential drowsiness they perceived. Furthermore, a thermal stimulation experiment was designed to explore the impact of tPBM-associated heat sensations on human EEG signals.

For the thermo_stim experiment, a heat stimulator (Pathway model ATS, Pain and Sensory Evaluation system, Medoc Advanced Medical Systems, Israel) was employed (see Fig. 2(a)) to 
$1438{ }^{\circ} \mathrm{C} / \mathrm{sec}$ for the alteration of temperature. The time-dependent temperature increases on the

144 subject's forehead induced by active tPBM were remeasured in this study using an infrared clinical

145 thermometer (Medical Head and Ear Thermometer, Metene, England) over several human

146 subjects; the group-averaged results were very consistent with those reported previously [14].

147

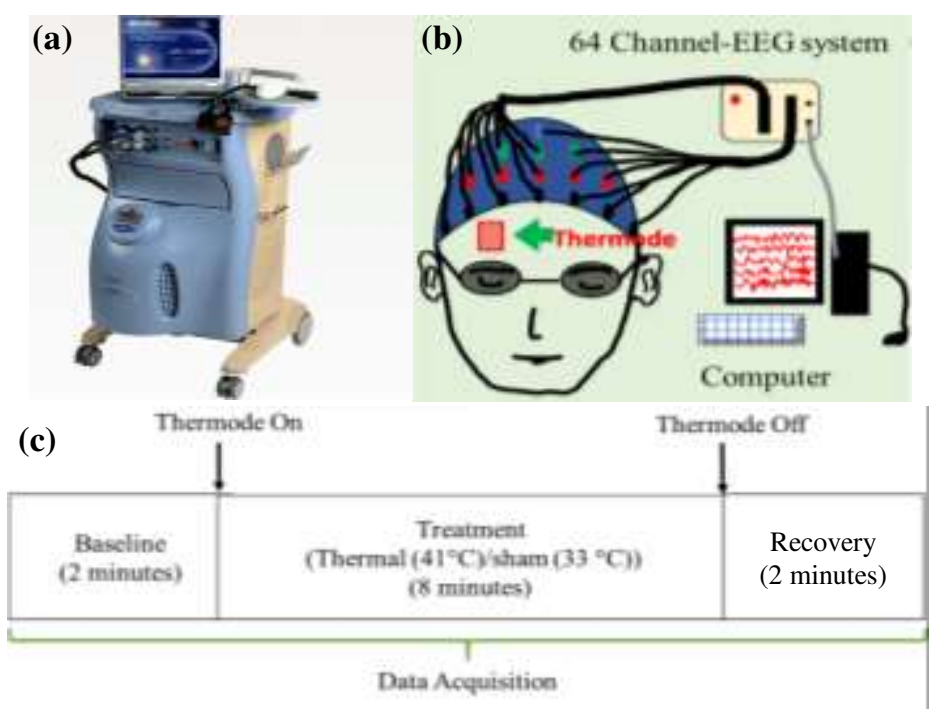

Fig. 2 Experimental setup and protocol for concurrent thermo_stim and EEG experiment. (a) Picture of the thermal simulator system (Pathway model with ATS thermode). (b) Schematic diagram of the experimental setup: 64 electrodes of an EEG device were attached to an international 10-10 standard EEG cap, while the subject retained eye-closed during the sham or active thermo_stim condition. The thermode was attached at the right forehead of the subject to produce heat mimicking that created in tPBM. (c) Shows the experimental protocol for thermal stimulation: including a 2-min baseline, 8-min thermo_stim, and a 2-min recovery period.

157 the tPBM was on the right forehead to simulate/mimic the thermal effect induced by tPBM, as 158 marked in Fig. 2(b). The same 64-channel EEG device (as used in the tPBM experiment) was 159 employed to concurrently record electrophysiological responses to the thermal stimulations. Fig. 160 2(c) shows the experimental protocol: It included a 2-min baseline and an 8-min thermal 161 stimulation, followed by a 2-min recovery period. The temperature of the thermal stimulator 
162 remained at $33^{\circ} \mathrm{C}$ during the 2-min baselines for both sham and active thermo_stim. For the active

163 stimulation, the temperature of the thermode increased from $33{ }^{\circ} \mathrm{C}$ to $41{ }^{\circ} \mathrm{C}$ following the tPBM-

164 equivalent thermal rate ${ }^{19}$ and was maintained at $41{ }^{\circ} \mathrm{C}$ during the remaining stimulation period.

165 Then we removed the thermode from the forehead during the 3-min recovery period. For the sham

166 experiment, we maintained the thermode's temperature at $33{ }^{\circ} \mathrm{C}$ throughout the 8 -min period

167 before removal of the thermode from the forehead while EEG recording lasted the entire 12-min

168 period. In either experiment, subjects were asked to keep their eyes closed throughout the whole 169 measurement time.

170 While the sampling rate of EEG during the thermo_stim experiment was $512 \mathrm{~Hz}$, we also 171 down sampled it to $256 \mathrm{~Hz}$ during data analysis to be consistent with that for the PBM experiment.

172 Further data analysis was performed only on the baseline and stimulation periods for the $173 \mathrm{tPBM} /$ sham and thermal/sham experiments, which accounts for the two minutes of baseline and

174 the eight minutes of stimulation.

\section{2.3. Data Analysis}

176 Each collected EEG dataset contained 64-channels of time series corresponding to the collected

177 large-scale neural activities at 64 scalp locations. Data from 3 participants from the tPBM group

178 were excluded due to drowsiness and fatigue from self-reporting and experimenter's observation.

179 Therefore, a total of 46 pairs (from 46 subjects) of EEG data under both sham and active tPBM 180 and a total of 14 pairs (from 14 subjects) of EEG under both sham and thermo_stim were included 181 for further data processing.

\section{$182 \quad$ 2.3.1. Data Preprocessing for EEG Time Series}

183 The data were preprocessed using MATLAB and a MATLAB-based, open-source software 184 package, EEGLAB. First, the acquired 64-channel EEG raw data were bandpass filtered at 0.5 - 
$18570 \mathrm{~Hz}$. A notch filter was applied to eliminate line noise at $60 \mathrm{~Hz}$. Re-referencing was performed

186 by subtracting the average of voltage signals across all the 64 electrodes from each of the EEG

187 time series. Second, robust principal component analysis and independent component analysis

188 were performed to remove artifacts, such as eye movements, saccades, and jaw clenches. Next,

189 64-electrode artifact-free time series were divided into three temporal segments: baseline, first 4

190 minutes (1-4 min) and last 4 minutes (5-8 min) during sham or tPBM stimulation. Specifically, (1)

191 the last 60 seconds of the 2-min baseline before the onset of tPBM/sham were used to quantify the

192 baseline signal as marked by $\mathrm{T}_{\text {base-tPBM_stim }}$ and $\mathrm{T}_{\text {base-tPBM_sham }}$, respectively; (2) the first 4 minutes

193 (1-4 min) of the tPBM/sham stimulation were labeled by $\mathrm{T}_{1-4-\mathrm{tPBM}}$ stim and $\mathrm{T}_{1-4-\mathrm{tPBM} \_ \text {sham }}$; and (3)

194 the second 4 minutes of the tPBM/sham stimulation were represented by $\mathrm{T}_{4-8 \text {-tPBM_stim }}$ and $\mathrm{T}_{4-8}$ -

195 tPBM_sham. The preprocessed data were then used to perform further analysis for each of the temporal

196 segments. Likewise, the EEG data under the thermo_stim condition were preprocessed following

197 the same steps as given above and then grouped and labeled into similar three temporal segments:

198 T $T_{\text {base-thermo_stim, }} \mathrm{T}_{\text {base-thermo_sham, }} \mathrm{T}_{\text {1-4-thermo_stim, }} \mathrm{T}_{\text {1-4-thermo_sham, }} \mathrm{T}_{4-8 \text {-thermo_stim, }}$ and $\mathrm{T}_{4-8-\text { thermo_sham, for }}$

199 both sham and active thermal stimulations.

200 2.3.2. EEG Power and Topography at Five Frequency Bands in Three Time Segments

201 For each time segment, every time series of 64 electrodes was bandpass filtered into five 202 conventional frequency bands: delta $(0.5-4 \mathrm{~Hz})$, theta $(4-8 \mathrm{~Hz})$, alpha $(8-13 \mathrm{~Hz})$, beta $(13-30 \mathrm{~Hz})$, 203 and gamma $(30-70 \mathrm{~Hz})$. The respective mean EEG powers at five frequency bands were achieved 204 with root-mean-square calculations over the chosen time segment using the MATLAB function 205 'rms' on each electrode of EEG. First, 2-sample t-tests were performed to verify that there was no 206 significant difference in EEG powers between the baselines of tPBM and its sham experiment (i.e., $207 P_{\text {base-tPBM_stim }}$ and $\left.P_{\text {base-tPBM_sham }}\right)$. In this way, we determined mean EEG power values for each 
208 time segment as $P_{1-4-t P B M_{-} \text {stim }}, P_{1-4-t P B M_{-} \text {sham }}, P_{4-8-t P B M_{-} \text {stim }}$, and $P_{4-8-t P B M_{-} \text {sham, }}$ respectively, for each 209 frequency band. To obtain changes from baseline, the EEG power at each frequency band during 210 the sham and active tPBM were normalized with respect to (i.e., being divided by) their own 1211 min baseline EEG powers during T $_{1-4-\text { tPBM_stim }}$ and T5-8-tPBM_stim $_{\text {for }}$ both sham and active tPBM 212 conditions, as expressed by four terms in eq. (1) below:

$$
\left(\frac{P_{1-4-t P B M} \text { sham }}{P_{\text {base-tPBM_sham }}}\right),\left(\frac{P_{5-8-t P B M_{-} s h a m}}{P_{\text {base-tPBM_sham }}}\right),\left(\frac{P_{1-4-t P B M_{-} s t i m}}{P_{\text {base-tPBM_stim }}}\right) \text {, and }\left(\frac{P_{5-8-t P B M} \text { _stim }}{P_{\text {base-tPBM_stim }}}\right) .
$$

This normalization process was repeated for all 64 electrodes, enabling us to form mean power 215 topographies during two periods of sham and active tPBM for each human subject. Consequently,

216 percentage topographies of sham-controlled, tPBM-induced changes in mean EEG power at each

217 frequency band $\left(\Delta P_{i-t P B M}^{f}\right.$ in $\left.\%\right)$ were obtained by subtracting the sham mean power values from 218 those under active tPBM at each electrode for both 1-4 min and 5-8 time periods, given as below:

$$
\Delta P_{i-t P B M}^{f}=\left(\frac{P_{i-t P B M_{\_} s t i m}^{f}}{P_{\text {base-tPBM_stim }}^{f}}-\frac{P_{i-t P B M_{\_} \text {sh }}^{f}}{P_{\text {base- } t P B M_{-} \text {sham }}^{f}}\right) \times 100 \%,
$$

220 where $i$ represents either 1-4 min or 5-8 min, and $f$ indicates different frequency bands.

221 Similarly, the same procedures of baseline normalization/ratios and sham-subtraction were 222 applied to the 64-channel EEG time series recorded in the thermo_stim experiments, producing 223 percentage topographies of sham-controlled changes in mean EEG power in response to thermal 224 stimulation at five frequency bands during the 1-4 min and 5-8 min stimulation periods for each 225 subject, as expressed by eq. (3):

$$
\Delta P_{i-\text { thermo_stim }}^{f}=\left(\frac{P_{i-\text { thermo_stim }}^{f}}{P_{\text {base-thermo_stim }}^{f}}-\frac{P_{i-\text { thermo_sham }}^{f}}{P_{\text {base-thermo_sham }}^{f}}\right) \times 100 \%,
$$

227 where $i$ represents either 1-4 min or 5-8 min, and $f$ marks different frequency bands. 


\subsubsection{Quantification of Net EEG Power Enhancements Induced by tPBM}

229 In theory, the measured percentage changes of sham-controlled mean EEG powers at respective

230 frequency bands, $\Delta P_{i-t P B M}^{f}$, should result from both the net tPBM effect, $\Delta P_{i-t P B M}^{f}(n e t)$, and

231 thermal effect, $\Delta P_{i-\text { thermo_stim }}^{f}$, namely,

$$
\Delta P_{i-t P B M}^{f}=\Delta P_{i-t P B M}^{f}(n e t)+\Delta P_{i-t h e r m o \_s t i m}^{f}
$$

233 where $i$ represents either 1-4 min or 5-8 min, and $f$ marks different frequency bands. Accordingly,

234 we would be able to determine net tPBM-induced EEG power changes (in \%) at respective

235 frequency bands, based on sham-controlled tPBM and thermo_stim EEG measurements (i.e., $236 \Delta P_{i-t P B M}^{f}$ and $\left.\Delta P_{i-t h e r m o \_s t i m}^{f}\right)$.

\section{2.3.4. Statistical Analysis}

238 Statistical analysis was performed to determine statistical significances of (1) sham-controlled 239 tPBM effects, (2) sham-controlled thermo_stim effects, and (3) the difference between sham240 controlled tPBM and sham-controlled thermal effects. For the first two statistical testing items,

241 paired t-tests were performed between percentage topographies of mean EEG power changes (i.e., 242 mPower in \%) under the sham and active tPBM as well as under the sham and thermo_stim, 243 respectively. Our cross-over designs for both tPBM and thermo_stim experiments justified the use 244 of paired t-tests. For the $3^{\text {rd }}$ statistical testing, two-sample t-tests were performed to identify 245 significant difference between the mPower values of sham-subtracted tPBM and sham-subtracted 246 thermal effects at each electrode site. Two-sample t-tests were chosen because of two different 247 groups of participants. Furthermore, the false discovery rate (FDR) corrections were performed 248 for comparison of topography to minimize type I errors in repeated t-tests among the 64 EEG 249 electrodes. All statistical tests were performed at $\alpha=0.05$ after FDR correction. 
Moreover, due to the unbalanced sample sizes between the tPBM and thermo_stim

251 experiments, the effect size $(d)$ at each electrode was calculated for the comparison between sham-

252 subtracted tPBM and sham-subtracted thermal stimulation. The " $d$ " is defined as the difference

253 between two means divided by the standard or pooled standard deviations of the two groups. In

254 general, $\mathrm{d}=0.2,0.5,0.8$, and 1.2 are considered a small, medium, large, and very large effect size,

255 respectively.

\section{3. Results}

257 We reported results based on several statistical comparisons. First, we looked at the sham-

258 controlled tPBM-induced effects on EEG powers at delta, theta, alpha, beta, and gamma bands.

259 Second, we investigated the sham-controlled thermo_stim effects on EEG powers at the same five

260 frequency bands. Last, we evaluated the differences between the sham-controlled tPBM versus 261 thermal effects for the respective frequency bands.

\section{3.1. Sham-controlled tPBM-induced Alterations in Mean EEG Power and Topography}

263 The top row of Fig. 3 illustrates the baseline-normalized, sham-subtracted topographies for EEG

264 percentage changes in mPower (i.e., \%) by tPBM at all five frequency bands averaged over $n=46$

265 participants. The second row of Fig. 3 shows statistical t-maps (obtained with paired t-tests and

266 FDR correction) during the first four minutes (T1-T4) and the second four minutes (i.e., T5-T8)

267 of the tPBM/sham stimulation for the respective frequencies.

268 The results clearly showed significantly decreased mPower at anterior and posterior cerebral

269 regions during the first 4-min of tPBM in the delta band. However, this decreased delta power was

270 not persistent after the first four minutes of tPBM. More important, significantly increased alpha

271 and beta mPower distributions were shown on multiple sites of the human scalp, particularly

272 during the second 4-min of tPBM. As seen in this figure, during the last half tPBM period, both 
273 the sham-controlled increase in mPower topography and t-map revealed anterior-posterior power-

274 enhancement patterns for the alpha band. A smaller number of significant increases in beta

275 mPower occurred, located at central and posterior regions of the scalp. No significant difference

276 in mPower was observed between tPBM and sham in theta and gamma bands.

277
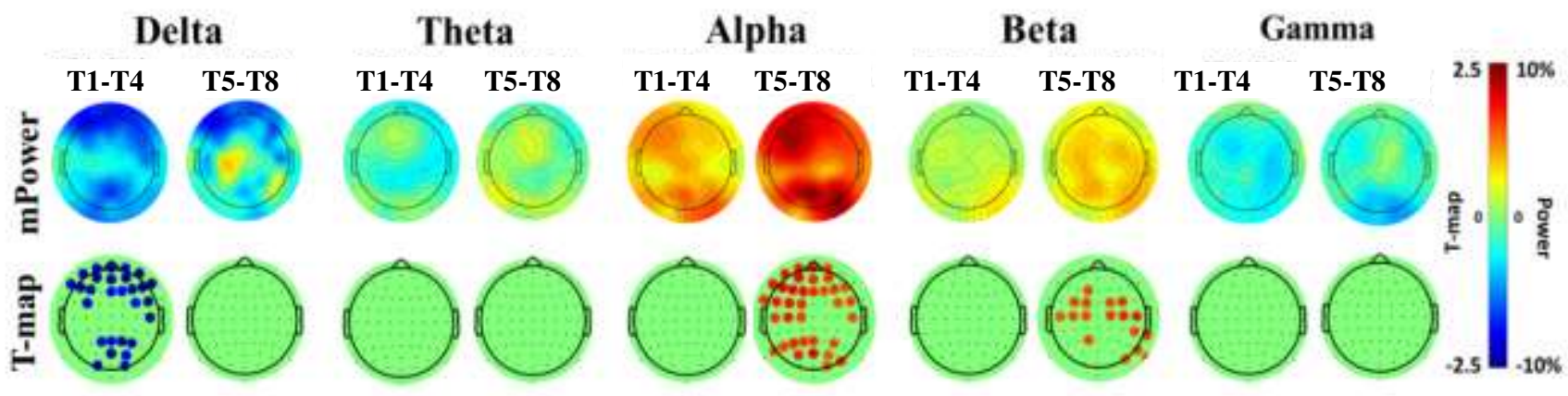

Fig. 3 Group-level ( $\mathrm{n}=46)$, baseline-normalized, sham-controlled topographies for EEG mean power (mPower) alterations induced by $\mathrm{tPBM}$. The first row shows the mean power changes during the first 4-min (T1-T4) and last 4-min (T5-T8) tPBM. The second row presents the corresponding statistical T-topographies for the comparison between tPBM versus sham stimulation, with paired t-tests at $\alpha=0.05$ after FDR correction for T1-T4 and T5-T8. Solid blue and red dots mark the sites that had significant changes in mPower with respect to the sham stimulations.

3.2. Sham-controlled Thermo_stim-induced Changes in Mean EEG Power and Topography

Following the same data presentation style as that in Fig. 3, The top row of Fig. 4 illustrates the baseline-normalized, sham-subtracted topographies for EEG mPower alterations (in \%) by

287 thermo_stim at all five frequency bands averaged over $n=14$ participants. The second row of Fig.

2884 shows statistical t-maps (obtained with paired t-tests and FDR correction) during the first and

289 second halves of the stimulation period. This figure clearly demonstrates that mean EEG powers

290 in alpha and beta frequencies were significantly reduced, particularly during the last half period of

291 thermal stimulation relative to the sham condition. Specifically, an anterior-posterior reduction 
took place in the alpha mPower while global decreases occurred in the beta mPower. However,

293 the delta, theta, and gamma bands did not show any significant changes in mPower.

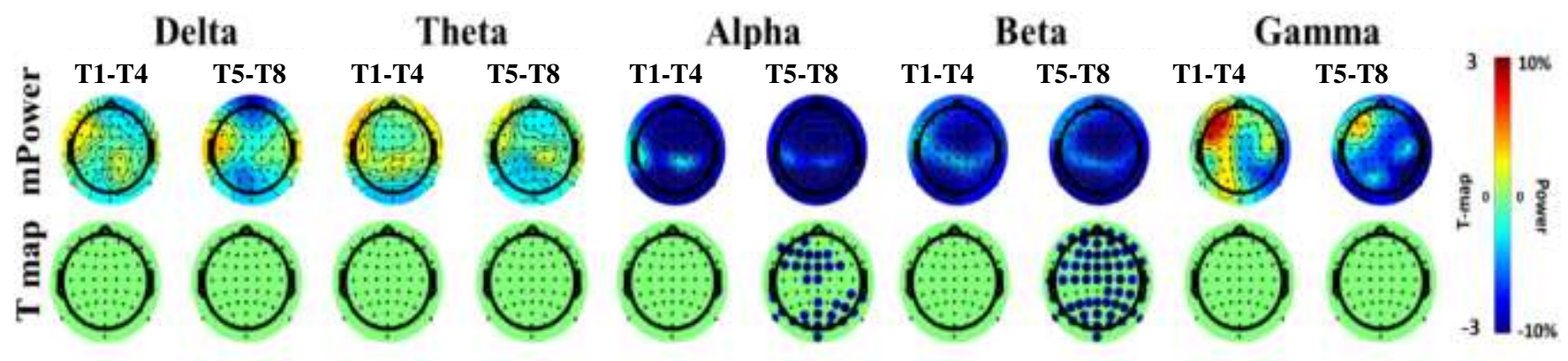

Fig. 4 Group-level $(n=14)$, baseline-normalized, sham-controlled topographies for EEG mean power (mPower) alterations induced by thermo_stim. The first row shows the mean power changes during the first 4-min (T1-T4) and last 4-min (T5-T8) tPBM. The second row presents the corresponding statistical T-topographies for the comparison between thermo_stim versus sham stimulation, with paired t-tests at $\alpha=0.05$ after FDR correction for T1-T4 and T5-T8. Solid blue dots mark the sites that have significant decreases in mPower with respect to the sham stimulations.

\subsection{Net EEG Power Enhancements Induced by tPBM Excluding Thermal Effects}

As expressed by eq. (4), we would be able to quantify net tPBM-induced EEG mPower increases at the group level by subtracting the sham-controlled thermo_stim mPower topography (top row of Fig. 4) from the sham-controlled tPBM mPower topography (i.e., top row of Fig. 3). This operation leads to Fig. 5(a), which illustrates the group-level difference of the baseline-normalized, sham-subtracted spatial distributions of mPower between tPBM and thermal stimulations, i.e., $\Delta P_{i-t P B M}^{f}(n e t)$, during $i=\mathrm{T} 1-\mathrm{T} 4$ and T5-T8 at $f=$ delta, theta, alpha, beta, and gamma bands. Figs. 5(b) and 5(c) are two statistical topographies for t-test (T-map) and effect sizes (ES) between shamcontrolled tPBM effects and sham-controlled thermal effects. The t-tests were based on 2-sample t-tests at $\alpha=0.05$ with FDR correction. 
311 patterns in alpha and beta bands with respect to those by tPBM. This opposite effect gave rise to a

312 larger difference between the two types of stimulations (i.e., $\Delta P_{i-t P B M}^{f}(n e t)$ ), as illustrated by Fig.

313 5(a), mainly at $f=$ alpha and beta bands. Statistically, the two-sample t-tests confirmed that the

314 alpha and beta mPower changes induced by tPBM were significantly higher with larger effect sizes

315 than those by thermo_stim at anterior-posterior regions during the entire 8-min stimulation period.

316 However, no significant photobiomodulation effects took place in delta, theta, and gamma bands.

Delta

(a)

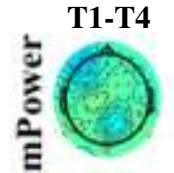

(b)

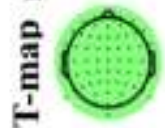

(c)

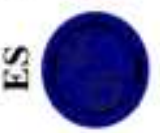

Theta
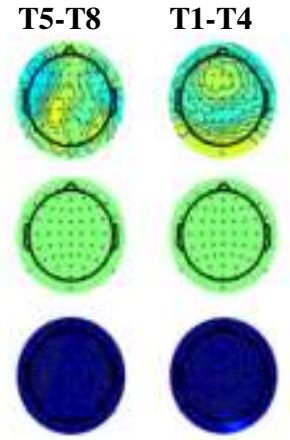
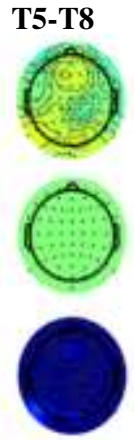

Alpha

Beta
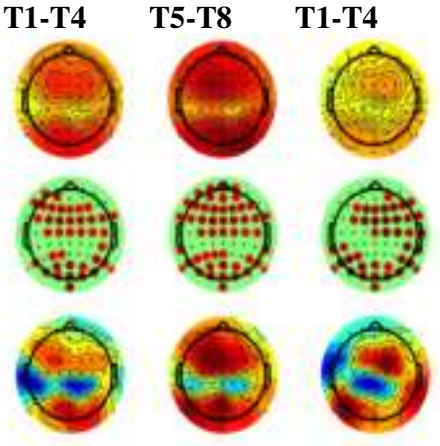
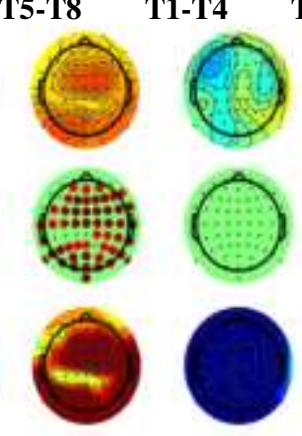

Gamma

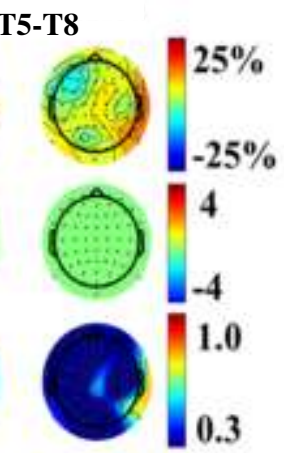

Fig. 5 (a) Group-level difference in mPower maps between baseline-normalized, sham-subtracted topographies from tPBM $(n=46)$ and thermo_stim $(n=14)$ experiments in all five frequency bands for the first 4-min (T1-T4) and second 4-min (T5-T8) of the stimulation period. (b) it shows statistical t-map topographies obtained from two-sample t-tests with red dots marking electrode sites having significant increases in ePower ( $\alpha=0.05$ with FDR correction) by tPBM with respect to thermo_stim. (c) Shows effect sizes (ES) for comparing tPBM and thermal effects.

\section{3}

\section{Discussion}

324 We recorded scalp EEG in vivo before, during, and after tPBM/sham from 49 human subjects

325 under the eyes-closed resting state. Furthermore, we generated the same temperature enhancement

326 induced by tPBM on human foreheads using a thermal generator. For the latter, we recorded EEG

327 in vivo from 14 human subjects before, during, and after the thermal stimulation with its 
328 corresponding sham experiment. Baseline-normalized EEG power alterations were compared

329 between tPBM laser-illumination and its sham experiments. More importantly, the sham-

330 subtracted, tPBM-induced EEG mPower topographies from 46 participants were compared at the

331 group level with the sham-subtracted thermal-induced EEG mPower taken from another group of

33214 human subjects. In this way, we rigorously investigated (1) how tPBM modulates brain rhythm

333 powers under the eyes-closed resting state, (2) whether and how thermal stimulation modulates

334 brain rhythm powers under the eyes-closed resting state, and (3) whether thermal stimulation effect

335 could be removed to recover/obtain net tPBM effects on the brain rhythm powers.

\section{4.1. tPBM-induced Alterations in EEG mPower at Alpha, Beta, and Delta Bands}

337 Fig. 3 illustrates the sham-controlled topographic mPower increments induced by tPBM laser-

338 illumination under eyes-closed resting state. During the last half period of tPBM, significantly

339 enhanced power values were clearly observed in the anterior-posterior regions for alpha

340 oscillations and in the central and posterior regions for beta oscillations. Overall, these results are

341 in good agreements with those taken under eyes-open resting state, as we reported before [15, 16].

342 While the alpha power is believed to be related to wakefulness ${ }^{28}$, it is also commonly

343 associated with cognition-related brain functions such as memory encoding, attention, and brain

344 network synchronization and interaction ${ }^{29-31}$. Moreover, studies indicate that cortical alpha waves

345 are engendered due to the collaboration of thalamocortical and cortico-cortical interactions ${ }^{32}$. With

346 several hundred human subjects, previous studies have demonstrated that 1064-nm laser enabled

347 significant behavioral improvements in cognitive functions using the same experimental protocol

$348 \quad 3,9-11,33$. Putting all these results together, we speculate that improvement of human cognition by

349 tPBM may be closely associated with alpha power increases and potential stimulation to the 
anterior-posterior network, which is an executive network that assists rapid instantiation of new

351 tasks by interacting with other control and processing networks ${ }^{34}$.

352 In addition, the literature has shown that beta activation is responsible for maintaining 353 concentration or focus. Several studies have presented evidence that improved beta waves are a 354 sign of better cognitive capacity ${ }^{35,36}$. Hostovecky et al. demonstrated that beta waves are essential 355 to neurofeedback activities ${ }^{35}$. Another study demonstrated that beta activation is an indication of 356 cognitive processes with higher concentration by conducting rejection tasks or mental arithmetic 357 tasks, both of which require attention and internal processing ${ }^{36}$. On the other hand, since beta 358 waves are a sign of somatosensory processing, beta activation might be responsible for the heat 359 sensation from the tPBM laser ${ }^{37}$. In our case, however, the beta response to heat would most 360 probably be limited to the left central cortex near the somatosensory region because the right 361 forehead was illuminated by the tPBM laser ${ }^{38}$. Thus, thermal sensation would not account for the 362 observation of beta activation beyond the somatosensory area (Fig. 3). There must exist another 363 mechanism of action for beta mPower increase, as discussed in Section 4.5.

364 Besides increasing alpha and beta mPower, tPBM reduced delta power during the first 4 365 minutes of stimulation. While the power of delta waves has been widely related to human sleep, 366 the power of delta oscillation at resting state has also been linked to cognitive functions. For 367 example, Bablioni et al. reported that better cognitive performance is associated with a decreased 368 power of delta waves ${ }^{39}$. During initial 4-min $\mathrm{tPBM}$, the significant decrease in delta power at 369 resting state might potentially enhance cognitive functions. Furthermore, according to the 370 literature, increased delta wave has been associated with fatigue ${ }^{40,41}$, depression ${ }^{42}$, and addiction 37143,44 . The modulatory effects of tPBM on delta waves are opposite to the abnormalities found in 372 patients with emotional and cognitive disorders. Thus, tPBM might have a promising prospect to 
373 reverse the changes seen in various psychiatric and psychological conditions ${ }^{40-44}$. However, this

374 expectation needs to be further investigated with an optimal selection of stimulation length and

375 intervention designs because of time-dependent patterns in delta mPower changes.

\section{4.2. Alterations in EEG mPower by Thermo_stim Are Opposite to Alterations by tPBM}

377 As shown in Fig. 4, the thermal stimulation following the equivalent temperature rise given by 378 tPBM, induced significant decreases of global alpha and beta powers, meaning strong 379 desynchronizations of alpha and beta waves across the entire scalp. These observations are very 380 consistent with previous EEG studies using non-noxious thermal stimuli ${ }^{45}$ and noxious thermal

381 stimuli ${ }^{46,47}$. However, few EEG studies have reported effects of nonpainful thermal stimuli given 382 on the human head since most of thermal stimulation sites were on peripheral locations ${ }^{45,47-50}$. One 383 study on tonic pain using continuous EEG to predict subjective pain perception observed 384 significant decreases in alpha $(7-10 \mathrm{~Hz})$ power during the stimulation and suggested that this 385 decrease was due to an augmented activity of cortico-cortical and thalamocortical feedback loops $38{ }^{50}$. There are numerous EEG-based publications to investigate mechanisms of pain, but they are 387 beyond the focus of this study. The major observation and conclusion drawn from Figs. 3 and 4 388 were that the trends and topographic patterns of percentage changes in EEG mPower induced by 389 thermo_stim were opposite to those by tPBM. In other words, these results confirmed 390 unambiguously that the percentage changes in EEG mPower at alpha and beta bands by sham391 controlled 1064-nm tPBM could not stem from the thermal impact of the laser used in tPBM.

\section{4.3. Net tPBM Effects Excluding Thermal Effects}

393 Because of the opposite trends in percentage changes of EEG mPower between the two types of 394 stimulations, as large as $\sim 25 \%$ increases in alpha mPower (Fig. 5(a)) and large effect sizes of 0.8 395 or larger (Fig. 5(c)) were achieved in an anterior-posterior pattern under tPBM during the entire 8- 
min period relative to the sham. Furthermore, the two-sample t-tests (Fig. 5(b)) statistically proved

397 that the two stimulation methods by laser and heat created distinct changes of electrophysiology

398 in alpha and beta frequencies across most of the electrodes sites. This conclusion is in excellent

399 agreement with one of our previous studies, which presented that the thermal effect was

400 independent and opposite to the tPBM impact on cerebral hemodynamic oxygenation ([HbO]) and

401 metabolic oxidation ([CCO]) near the tPBM site ${ }^{19}$. Taking all these observations together

402 emphasized that the detected alterations in alpha and beta EEG mPower globally, [HbO] locally,

403 and [CCO] locally resulted from only 1064-nm tPBM, not from thermal sensation. The

404 enhancement of anterior-posterior synchronization by tPBM at alpha and beta waves might be due

405 to another mechanism or electrophysiological path, which will be explored and examined in our

406 future studies.

407 In addition, the laser/thermal stimulation applied in this study has been proved safe, non-

408 painful, and often little perceptible to human subjects at the laser power of $\sim 250 \mathrm{~mW} / \mathrm{cm}^{2}$. A study

409 conducted on a rabbit brain using CW and pulsed lasers demonstrated that the heat generated by a

410 laser with less than $750 \mathrm{~mW} / \mathrm{cm}^{2}$ does not cause tissue damage ${ }^{51}$.

\section{4.4. tPBM-induced EEG mPower Changes in Eyes-open and Eyes-closed Resting State}

412 One of the objectives of this study was to compare the tPBM-induced effects between eyes-open

413 and eyes-closed resting state because EEG signals have been shown to behave differently between

414 these states ${ }^{26,52}$. In the eyes-open resting state, the human brain encounters many visual stimuli

415 and activates the visual information processing networks/paths. However, those processes and

416 pathways are suppressed during the eyes-closed resting state due to the blockage of visual

417 information input ${ }^{52}$, indicating the distinct emphasis of brain networks and processes in these two 
418 states. This is why the eyes-closed state naturally creates higher absolute powers of alpha wave 419 compared to the eyes-open resting state on healthy humans ${ }^{52}$.

420 However, the abovementioned difference between the eyes-open and eyes-closed state would 421 not necessarily affect the comparisons of the tPBM-induced EEG mPowers at alpha and other 422 frequency bands between the two states in the current study because the comparisons were taken 423 after baseline normalization and sham subtraction for each participant. In this way, the sham424 controlled, tPBM-evoked mPowers under the eyes-closed state given in Fig. 3 can be fairly 425 compared with those under the eyes-open state as reported in Ref. ${ }^{20}$. Indeed, they both show very 426 agreeable trends and patterns of alterations of mPowers at both alpha and beta bands. Moreover, 427 the increases in alpha/beta mPower are also compatible with several other published tPBM studies. 428 For example, using an 810-nm pulsed LED system, Zomorrodi et al. reported reduction in delta 429 power and improvement in alpha and beta power after 20-min PBM $^{24}$. These results emphasized 430 the robustness of alpha and beta waves neuromodulation by tPBM.

431 Nevertheless, there were a couple of slight differences in the global distribution of power 432 changes between the two resting states. For example, tPBM resulted in more beta power activation 433 in the central regions with the eyes-closed state (Fig. 3) and in the occipital areas with eyes-open 434 state [15]. Moreover, significant deactivation in the delta power was not observed in the eyes-open 435 experiment [15]. Such differences might have resulted from subtle differences between the 436 experimental protocols. In our previous eyes-open study, the stimulation was 11 min with 2.2-W 437 optical power for tPBM delivered by the same laser. In the current study, to minimize the 438 possibility of subjects being drowsy during the experiment, we designed an 8-min stimulation at $4393.5 \mathrm{~W}$, with the same total optical energy as in the 11-min tPBM study. The current study utilized 440 a little higher laser power compared to the previous one, possibly leading to more heat/warm 
441 sensation by the participants. As shown in Fig. 4, the cross-subject topographies of thermal

442 stimulation may reveal potential thermal sensation because of the reduced delta power at the frontal

443 region.

444 4.5. Methods of EEG Power Analysis, Post-Stimulation, and Frontoparietal Network

445 We employed the root-mean-square (RMS) method to quantify EEG powers during three separate

446 temporal segments (i.e., 2-min baseline, 1-4 min, and 5-8 min during tPBM) at each of the five

447 frequency bands. Conventionally, EEG powers are quantified by converting the time-domain data

448 to the frequency-domain power spectrum density (PSD) via Fourier transform, followed by

449 spectral average within the selected spectral range. Indeed, we have tested the results using both

450 RMS and PSD for several EEG time series under sham and active tPBM; the results confirmed

451 that the outcomes from both methods were in excellent agreement, as shown in supplementary

452 material, Fig. S1.

453 In this study, we did not present post-stimulation results in either tPBM or thermo_stim

454 experiments. Our focus was to address (1) whether there existed any thermal impact, created by

455 1064-nm tPBM equivalent, on the changes of EEG powers at five frequency bands, and (2)

456 whether the tPBM-evoked net increases in EEG powers at alpha and beta waves were unaffected

457 after removal of the thermal impact on EEG powers. Thus, it was not necessary to inspect results

458 in the post-stimulation period.

459 Overall, our results presented strong and significant enhancement by tPBM in EEG power or 460 synchronization for frontal-parietal alpha and beta oscillations. It is acknowledged ${ }^{34}$ that the 461 frontoparietal network is a flexible hub for cognitive control and "a distinct control network, in 462 part functioning to flexibly interact with and alter other functional brain networks. This network 463 coordination likely occurs in a $4 \mathrm{~Hz}$ to $73 \mathrm{~Hz} \theta / \alpha$ rhythm, both during resting state and task state." 
464 Thus, it is reasonable to speculate that the ability of tPBM to strongly modulate or synchronize

465 alpha and beta oscillations in the frontoparietal network may be closely associated with or serves

466 as the electrophysiological mechanism of action that IPBM is able to significantly improve human

467 cognition observed by our group [3, 7-9] and others $4,7,8,24$.

468 Moreover, according to ${ }^{34}$, "precision mapping of individual human brains has revealed that

469 the functional topography of the frontoparietal network is variable between individuals, 470 underscoring the notion that group-average studies of the frontoparietal network may be obscuring 471 important typical and atypical features." This notion explains why the observed spatial distribution

472 of enhanced EEG alpha and beta mPower was rather spread across frontal-parietal regions, in 473 addition to a systematic backwards shift of the EEG cap.

\section{4.6. Limitations and Future Work}

475 This study also had several drawbacks and thus have opportunities for future work. First, the 476 thermal stimulation was given based on contact delivery from the thermode to the human forehead, 477 whereas equivalent heat emitted from the laser in tPBM was non-contact. Also, the total area of 478 thermal stimulation by the thermode was relatively smaller than the site of tPBM laser aperture. 479 Second, the two sample sizes for tPBM and thermo_stim experiments were too unbalanced with 480 the thermal group having too fewer participants $(n=14)$, which may cause inaccurate or insufficient 481 statistical conclusions. Last, the international 10-10 EEG cap system in this study was not strictly 482 followed since a clear area with 4-cm in diameter was needed for tPBM light delivery on the right 483 forehead. Thus, the 64-channel EEG cap was shifted about 1-2 cm backwards, which would create $484 \sim 1-2 \mathrm{~cm}$ location errors in standard 64-channel topographies given in Figs. 3-5. As for future work, 485 a non-contact heat generator may be a better option to replicate the findings in this study with a 486 larger number of human subjects. To obtain/mark more accurate mPower topography with correct 
487 electrode locations, a 3-dimensional digitizer can be utilized to quantify exact locations or 488 coordinates of the 64 electrodes with respect to those in the 10-10 system on each subject's head, 489 followed by modification or correction of the EEG power topography using interpolation and 490 extrapolation based on the standard 10-10 electrode system. Last, more quantitative analysis on 491 network connectivity and directional information flow will be taken to substantiate our expectation 492 that $\mathrm{PBM}$ indeed modulates the frontoparietal network significantly during and post tPBM.

\section{5. Conclusion}

494 This study demonstrated that baseline-normalized, sham-controlled tPBM with a 1064-nm laser 495 given on the right forehead of healthy human subjects neuromodulated delta, alpha, and beta 496 oscillations in eyes-closed resting state. Moreover, we demonstrated that thermal stimulations

497 would generate opposite percentage changes in alpha and beta oscillation powers with respect to 498 those by tPBM. After careful two-sample t-tests, we proved our hypothesis that tPBM-induced 499 increases in anterior-posterior EEG powers at alpha and beta bands remained statistically 500 significant during the eyes-closed resting state, similar to those during the eyes-open resting state, 501 after removal of tPBM-associated thermal effects. The observed strong enhancement by tPBM on 502 alpha and beta oscillations in the anterior-posterior regions may be the underlying 503 electrophysiological mechanism of action to explain why tPBM enables to improve human 504 cognition. 


\section{Acknowledgments}

507 This work was supported in part by the National Institute of Mental Health/National Institutes of

508 Health under the BRAIN Initiative (RF1MH114285).

\section{Author contributions}

510 Drs. Wanniarachchi, Wang, and Liu designed the experimental protocol. Drs. Wanniarachchi,

511 Wang, and Wu conducted the study, including human subject recruitment, instrument setup and

512 calibration, data collection, and data analysis. Drs. Wanniarachchi and Wang prepared the

513 manuscript draft with important intellectual input and supervision from Dr. Liu. Drs. Liu and

514 Gonzalez-Lima edited and finalized the manuscript. All authors approved the final manuscript.

515 The National Institute of Mental Health provided funding for the study to Drs. Liu and Gonzalez516 Lima.

\section{Competing interests}

519 The authors declare no competing interests.

\section{Reference}

5211 Chung, H. et al. The nuts and bolts of low-level laser (light) therapy. Ann Biomed Eng 40, 516-533, doi:10.1007/s10439-011-0454-7 (2012). Functionally Inactivated by Toxins: ROLE OF CYTOCHROME c OXIDASE. Journal of Biological Chemistry 280, 4761-4771 (2005).

4 Hamblin, M. R. in Photobiomodulation in the Brain (eds M. R. Hamblin \& Y. Y. Huang) (Academic Press, 2019).

5 Rojas, J. C. \& Gonzalez-Lima, F. Neurological and psychological applications of $\begin{array}{lll}534 & 7 & \text { Naeser, M. A., Saltmarche, A., Krengel, M. H., Hamblin, M. R. \& Knight, J. A. Improved } \\ 535 & \text { cognitive function after transcranial, light-emitting diode treatments in chronic, traumatic }\end{array}$ transcranial lasers and LEDs. Biochem Pharmacol 86, 447-457, doi:10.1016/j.bcp.2013.06.012 (2013).

6 Cassano, P. in 2018 Conference on Lasers and Electro-Optics (CLEO). 1-2. brain injury: two case reports. Photomed Laser Surg 29, 351-358, doi:10.1089/pho.2010.2814 (2011). 
5388 Naeser, M. A. et al. Significant improvements in cognitive performance post-transcranial, 539 red/near-infrared light-emitting diode treatments in chronic, mild traumatic brain injury: $540 \quad$ open-protocol study. J Neurotrauma 31, 1008-1017, doi:10.1089/neu.2013.3244 (2014).

5419 Barrett, D. W. \& Gonzalez-Lima, F. Transcranial infrared laser stimulation produces beneficial cognitive and emotional effects in humans. Neuroscience 230, 13-23, doi:10.1016/j.neuroscience.2012.11.016 (2013).

Blanco, N. J., Saucedo, C. L. \& Gonzalez-Lima, F. Transcranial infrared laser stimulation improves rule-based, but not information-integration, category learning in humans. $\begin{array}{lllll}\text { Neurobiology of Learning and Memory } & \text { 139, }\end{array}$ doi:https://doi.org/10.1016/j.nlm.2016.12.016 (2017).

11 Blanco, N. J., Maddox, W. T. \& Gonzalez-Lima, F. Improving executive function using transcranial infrared laser stimulation. Journal of Neuropsychology 11, 14-25, doi:10.1111/jnp.12074 (2017).

12 Vargas, E. et al. Beneficial neurocognitive effects of transcranial laser in older adults. Lasers Med Sci 32, 1153-1162, doi:10.1007/s10103-017-2221-y (2017).

13 O'Donnell, C. M., Barrett, D. W., Fink, L. H., Garcia-Pittman, E. C. \& Gonzalez-Lima, F. Transcranial Infrared Laser Stimulation Improves Cognition in Older Bipolar Patients: Proof of Concept Study. J Geriatr Psychiatry Neurol, 891988720988906, doi:10.1177/0891988720988906 (2021).

14 Wang, X. et al. Up-regulation of cerebral cytochrome-c-oxidase and hemodynamics by transcranial infrared laser stimulation: A broadband near-infrared spectroscopy study. $J$ Cereb Blood Flow Metab 37, 3789-3802, doi:10.1177/0271678X17691783 (2017).

Wu, Q., Wang, X., Liu, H. \& Zeng, L. Learning Hemodynamic Effect of Transcranial and Health Informatics, 1-1, doi:10.1109/JBHI.2019.2951772 (2019).

16 Pruitt, T. et al. Transcranial Photobiomodulation (tPBM) With 1,064-nm Laser to Improve Cerebral Metabolism of the Human Brain In Vivo. Lasers Surg Med, doi:10.1002/lsm.23232 (2020).

17 Karu, T. Primary and secondary mechanisms of action of visible to near-IR radiation on cells. J Photochem Photobiol B 49, 1-17, doi:10.1016/S1011-1344(98)00219-X (1999).

18 Rojas, J. C. \& Gonzalez-Lima, F. Low-level light therapy of the eye and brain. Eye Brain 3, 49-67, doi:10.2147/EB.S21391 (2011).

19 Wang, X. et al. Impact of heat on metabolic and hemodynamic changes in transcranial infrared laser stimulation measured by broadband near-infrared spectroscopy. $N P h \mathbf{5}$, 011004, doi:10.1117/1.NPh.5.1.011004 (2017).

20 Wang, X. et al. Transcranial photobiomodulation with 1064-nm laser modulates brain electroencephalogram rhythms. NPh 6, 1, doi:10.1117/1.NPh.6.2.025013 (2019).

21 Wang, X., Dmochowski, J., Husain, M., Gonzalez-Lima, F. \& Liu, H. Proceedings \#18. Transcranial Infrared Brain Stimulation Modulates EEG Alpha Power. Brain Stimulation 10, e67-e69, doi:https://doi.org/10.1016/j.brs.2017.04.111 (2017).

22 Spera, V. et al. Transcranial Near-Infrared Light: Dose-Dependent Effects on EEG Oscillations but not Cerebral Blood Flow. bioRxiv, 837591, doi:10.1101/837591 (2019).

23 Berman, M. H., Hamblin, M. R. \& Chazot, P. in Rhythmic Stimulation Procedures in Neuromodulation (eds James R. Evans \& Robert P. Turner) 97-129 (Academic Press, 2017). 
24 Zomorrodi, R., Loheswaran, G., Pushparaj, A. \& Lim, L. Pulsed Near Infrared Transcranial and Intranasal Photobiomodulation Significantly Modulates Neural Oscillations: a pilot exploratory study. Sci Rep 9, 6309, doi:10.1038/s41598-019-42693-x (2019).

25 Yao, Y., Lian, Z., Liu, W. \& Shen, Q. Experimental study on physiological responses and thermal comfort under various ambient temperatures. Physiology \& Behavior 93, 310-321, doi:https://doi.org/10.1016/j.physbeh.2007.09.012 (2008).

26 Barry, R. J., Clarke, A. R., Johnstone, S. J., Magee, C. A. \& Rushby, J. A. EEG differences between eyes-closed and eyes-open resting conditions. Clin Neurophysiol 118, 2765-2773, doi:10.1016/j.clinph.2007.07.028 (2007).

27 Wang, X., Tian, F., Soni, S. S., Gonzalez-Lima, F. \& Liu, H. Interplay between upregulation of cytochrome-c-oxidase and hemoglobin oxygenation induced by near-infrared laser. Scientific Reports 6, 30540, doi:10.1038/srep30540 (2016).

28 Cantero, J. L., Atienza, M. \& Salas, R. M. Human alpha oscillations in wakefulness, drowsiness period, and REM sleep: different electroencephalographic phenomena within the alpha band. Neurophysiol Clin 32, 54-71, doi:10.1016/s0987-7053(01)00289-1 (2002).

29 Palva, S. \& Palva, J. M. New vistas for alpha-frequency band oscillations. Trends Neurosci 30, 150-158, doi:10.1016/j.tins.2007.02.001 (2007).

30 Hanslmayr, S., Gross, J., Klimesch, W. \& Shapiro, K. L. The role of alpha oscillations in temporal attention. Brain Res Rev 67, 331-343, doi:10.1016/j.brainresrev.2011.04.002 (2011).

31 Klimesch, W. EEG-alpha rhythms and memory processes. Int J Psychophysiol 26, 319340, doi:10.1016/s0167-8760(97)00773-3 (1997).

32 Steriade, M., Gloor, P., Llinas, R. R., Lopes de Silva, F. H. \& Mesulam, M. M. Report of IFCN Committee on Basic Mechanisms. Basic mechanisms of cerebral rhythmic activities. Electroencephalogr Clin Neurophysiol 76, 481-508, doi:10.1016/0013-4694(90)90001-z (1990).

33 Hwang, J., Castelli, D. M. \& Gonzalez-Lima, F. Cognitive enhancement by transcranial laser stimulation and acute aerobic exercise. Lasers Med Sci 31, 1151-1160, doi:10.1007/s10103-016-1962-3 (2016).

34 Marek, S. \& Dosenbach, N. U. F. The frontoparietal network: function, electrophysiology, and importance of individual precision mapping. Dialogues Clin Neurosci 20, 133-140 (2018).

35 Host'ovecký, M. \& Babušiak, B. Brain activity: beta wave analysis of 2D and 3D serious games using EEG. Journal of Applied Mathematics, Statistics and Informatics 13, 39-53, doi:https://doi.org/10.1515/jamsi-2017-0008 (2017).

36 Ray, W. J. \& Cole, H. W. EEG alpha activity reflects attentional demands, and beta activity reflects emotional and cognitive processes. Science 228, 750-752, doi:10.1126/science.3992243 (1985).

37 Breton, A. et al. Identifying the neural signature of thermic comfort sensation: neuroergonomic evaluation of a new ventilating system integrated in car seat. Human Neuroscience Archive, doi:10.3389/conf.fnhum.2018.227.00008.

38 Olausson, H. et al. Cortical activation by tactile and painful stimuli in hemispherectomized patients. Brain 124, 916-927, doi:10.1093/brain/124.5.916 (2001).

39 Babiloni, C. et al. Resting state cortical electroencephalographic rhythms are related to gray matter volume in subjects with mild cognitive impairment and Alzheimer's disease. Hum Brain Mapp 34, 1427-1446, doi:10.1002/hbm.22005 (2013). 
62940 Lal, S. K. \& Craig, A. Driver fatigue: electroencephalography and psychological 630 assessment. Psychophysiology 39, 313-321, doi:10.1017/s0048577201393095 (2002).

63141 Lal, S. K. \& Craig, A. Reproducibility of the spectral components of the electroencephalogram during driver fatigue. Int $J$ Psychophysiol 55, 137-143, doi:10.1016/j.ijpsycho.2004.07.001 (2005). Knyazev, G. G. EEG delta oscillations as a correlate of basic homeostatic and motivational processes. Neuroscience \& Biobehavioral Reviews 36, 677-695 (2012). Shufman, E. et al. Electro-encephalography spectral analysis of heroin addicts compared with abstainers and normal controls. Isr J Psychiatry Relat Sci 33, 196-206 (1996).

4 Synytsky, V. et al. Neurophysiological and psychophysiological effects of sulpiride in the norm and in patients with opiate addiction. Neurophysiology 34, 313-320 (2002).

45 Lv, B., Su, C., Yang, L. \& Wu, T. Effects of stimulus mode and ambient temperature on cerebral responses to local thermal stimulation: An EEG study. Int J Psychophysiol 113, 17-22, doi:10.1016/j.ijpsycho.2017.01.003 (2017).

47 Huishi Zhang, C., Sohrabpour, A., Lu, Y. \& He, B. Spectral and spatial changes of brain rhythmic activity in response to the sustained thermal pain stimulation. Hum Brain Mapp 37, 2976-2991, doi:10.1002/hbm.23220 (2016).

8 Bromm, B. \& Lorenz, J. Neurophysiological evaluation of pain. Electroencephalogr Clin Neurophysiol 107, 227-253, doi:10.1016/s0013-4694(98)00075-3 (1998).

49 Kazarians, H., Scharein, E. \& Bromm, B. Laser evoked brain potentials in response to painful trigeminal nerve activation. International Journal of Neuroscience 81, 111-122, doi:10.3109/00207459509015303 (1995).

50 Nir, R.-R., Sinai, A., Moont, R., Harari, E. \& Yarnitsky, D. Tonic pain and continuous EEG: Prediction of subjective pain perception by alpha-1 power during stimulation and at rest. Clinical Neurophysiology 123, 605-612, doi:10.1016/j.clinph.2011.08.006 (2012).

51 Chen, Y., De Taboada, L., O’Connor, M., Delapp, S. \& Zivin, J. A. Thermal effects of transcranial near-infrared laser irradiation on rabbit cortex. Neuroscience Letters 553, 99103, doi:https://doi.org/10.1016/j.neulet.2013.07.049 (2013).

52 Chen, A. C., Feng, W., Zhao, H., Yin, Y. \& Wang, P. EEG default mode network in the human brain: spectral regional field powers. Neuroimage 41, 561-574, doi:10.1016/j.neuroimage.2007.12.064 (2008). 
Figures

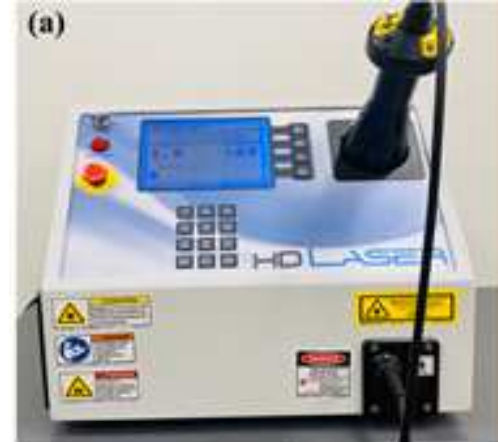

(c) Laser On

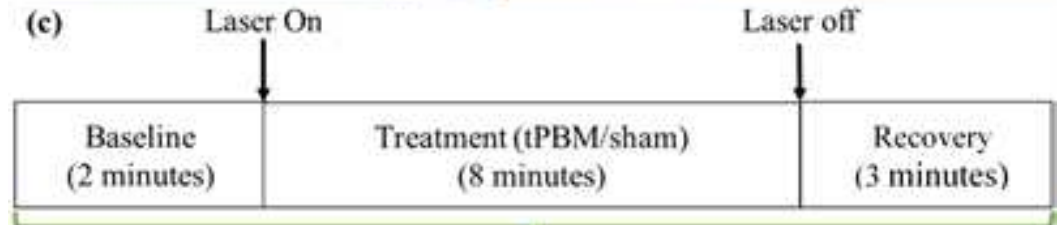

(b) 64-Channel EEG system

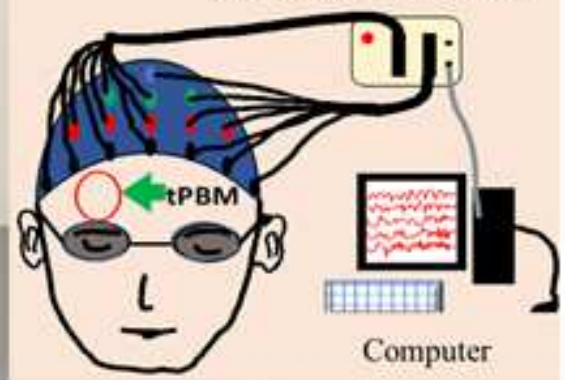

Computer

Data Acquisition

Figure 1

Experimental setup for concurrent tPBM and EEG experiment. (a) Photo of FDA-cleared, 1064-nm, continuous-wave laser (Model CG-500). (b) Schematic diagram of the experimental setup: 64 electrodes of an EEG device were attached to an international 10-10 standard EEG cap, while the subject was wearing a pair of safety goggles and retained in eye-closed resting state during the sham or active tPBM condition. The laser aperture was pointed at the right forehead of the subject with 2-cm away from the skin. (c) Shows the experimental protocol including a 2-min baseline, 8-min stimulation (with a laser power of $3.5 \mathrm{~W}$ for tPBM and $0 \mathrm{~W}$ for sham), and a 3-min recovery period.

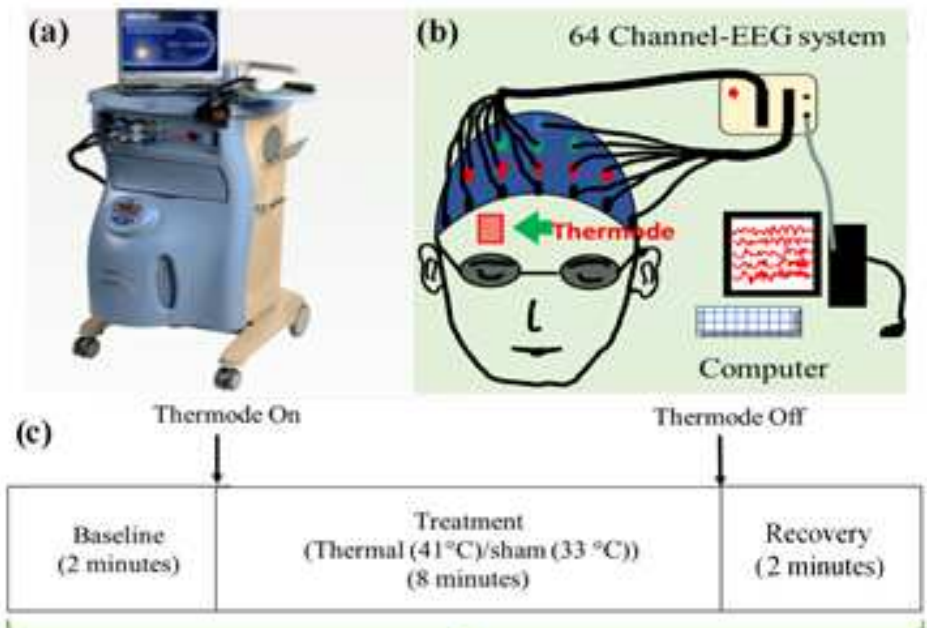

Data Acquisition

Figure 2 
Experimental setup and protocol for concurrent thermo_stim and EEG experiment. (a) Picture of the thermal simulator system (Pathway model with ATS thermode). (b) Schematic diagram of the experimental setup: 64 electrodes of an EEG device were attached to an international 10-10 standard EEG cap, while the subject retained eye-closed during the sham or active thermo_stim condition. The thermode was attached at the right forehead of the subject to produce heat mimicking that created in tPBM. (c) Shows the experimental protocol for thermal stimulation: including a 2-min baseline, 8-min thermo_stim, and a 2-min recovery period.

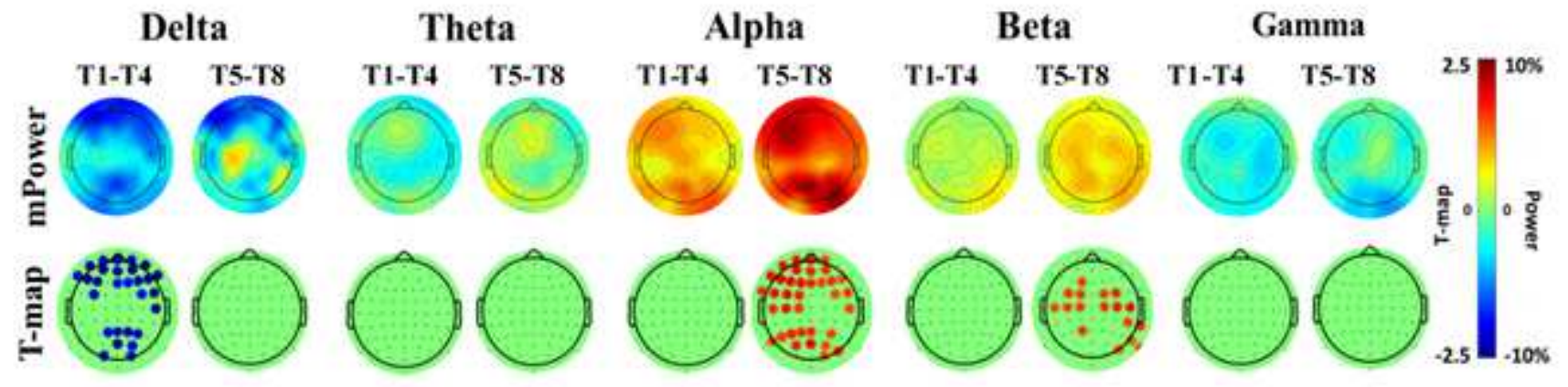

Figure 3

Group-level $(n=46)$, baseline-normalized, sham-controlled topographies for EEG mean power (mPower) alterations induced by tPBM. The first row shows the mean power changes during the first 4-min (T1-T4) and last 4-min (T5-T8) tPBM. The second row presents the corresponding statistical T-topographies for the comparison between tPBM versus sham stimulation, with paired t-tests at $\alpha=0.05$ after FDR correction for T1-T4 and T5-T8. Solid blue and red dots mark the sites that had significant changes in mPower with respect to the sham stimulations.

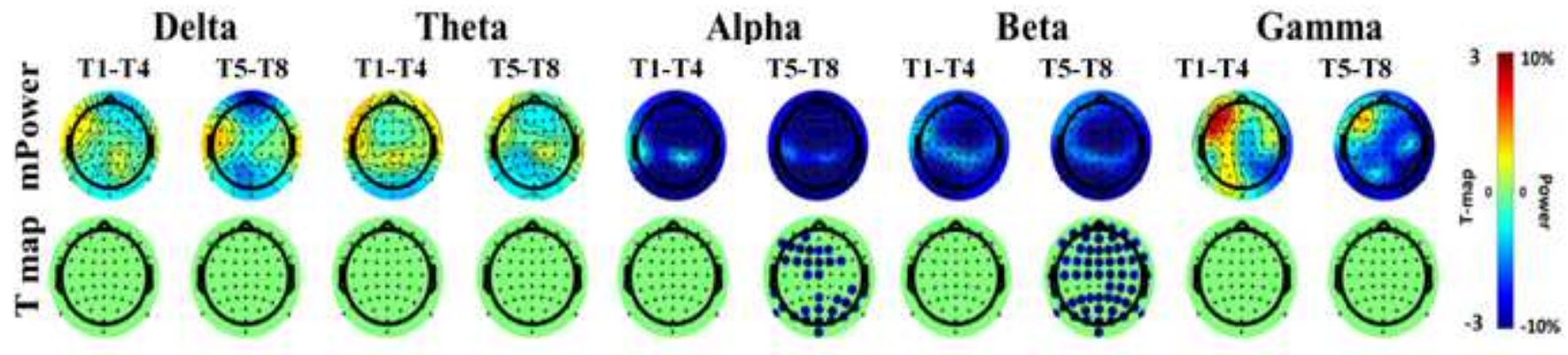

\section{Figure 4}

Group-level ( $n=14)$, baseline-normalized, sham-controlled topographies for EEG mean power (mPower) alterations induced by thermo_stim. The first row shows the mean power changes during the first 4-min (T1-T4) and last 4-min (T5-T8) tPBM. The second row presents the corresponding statistical Ttopographies for the comparison between thermo_stim versus sham stimulation, with paired t-tests at $\mathrm{a}=$ 0.05 after FDR correction for T1-T4 and T5-T8. Solid blue dots mark the sites that have significant decreases in mPower with respect to the sham stimulations. 
Delta

(a)

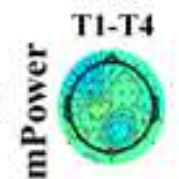

(b)

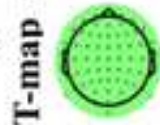

(c)

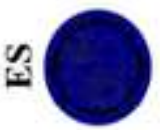

Theta
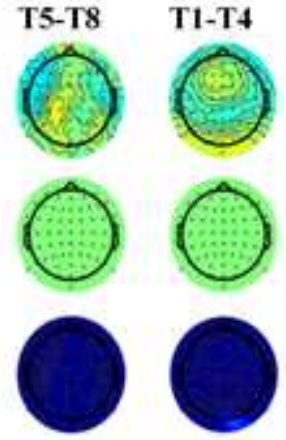

Alpha

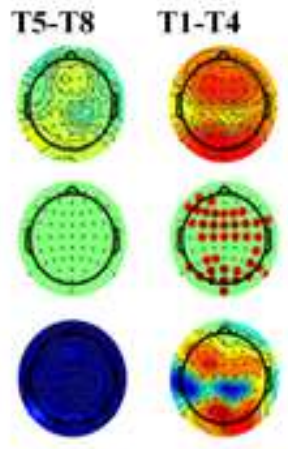

Beta
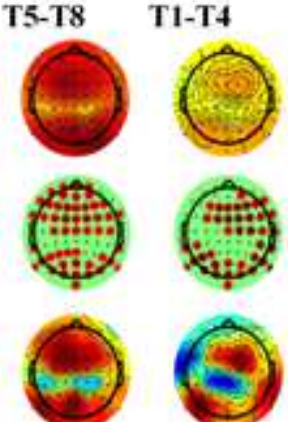

Gamma

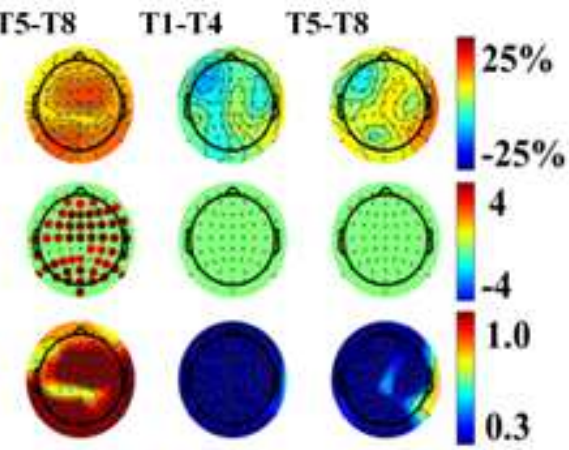

Figure 5

(a) Group-level difference in mPower maps between baseline-normalized, sham-subtracted topographies from tPBM $(n=46)$ and thermo_stim $(n=14)$ experiments in all five frequency bands for the first 4-min (T1T4) and second 4-min (T5-T8) of the stimulation period. (b) it shows statistical t-map topographies obtained from two-sample t-tests with red dots marking electrode sites having significant increases in ePower ( $a=0.05$ with FDR correction) by tPBM with respect to thermo_stim. (c) Shows effect sizes (ES) for comparing tPBM and thermal effects.

\section{Supplementary Files}

This is a list of supplementary files associated with this preprint. Click to download.

- Supplementaryfigurefinal.docx 